\title{
Minoxidil Promotes Hair Growth through Stimulation of Growth Factor Release from Adipose-Derived Stem Cells
}

\author{
Nahyun Choi ${ }^{1,2}$, Soyoung Shin ${ }^{3}$, Sun U. Song ${ }^{4, *}$ and Jong-Hyuk Sung ${ }^{1,2, *}$ \\ 1 College of Pharmacy, Yonsei University, Incheon 21983, Korea; nh147837@gmail.com \\ 2 STEMORE Co., Ltd., Incheon 21983, Korea \\ 3 College of Pharmacy, Wonkwang University, Iksan 54538, Jeonbuk, Korea; shins@wku.ac.kr \\ 4 Translational Research Center and Inha Research Institute for Medical Sciences, \\ Inha University School of Medicine, Incheon 21983, Korea \\ * Correspondence: sunuksong@inha.ac.kr (S.U.S.); brian99@empal.com (J.-H.S.); \\ Tel.: +82-32-890-2460 (S.U.S.); +82-32-749-4506 (J.-H.S.)
}

Received: 12 February 2018; Accepted: 26 February 2018; Published: 28 February 2018

\begin{abstract}
Minoxidil directly promotes hair growth via the stimulation of dermal papilla (DP) and epithelial cells. Alternatively, there is little evidence for indirect promotion of hair growth via stimulation of adipose-derived stem cells (ASCs). We investigated whether minoxidil stimulates ASCs and if increased growth factor secretion by ASCs facilitates minoxidil-induced hair growth. Telogen-to-anagen induction was examined in mice. Cultured DP cells and vibrissae hair follicle organ cultures were used to further examine the underlying mechanisms. Subcutaneous injection of minoxidil-treated ASCs accelerated telogen-to-anagen transition in mice, and increased hair weight at day 14 post-injection. Minoxidil did not alter ASC proliferation, but increased migration and tube formation. Minoxidil also increased the secretion of growth factors from ASCs, including chemokine (C-X-C motif) ligand 1 (CXCL1), platelet-derived endothelial cell growth factor (PD-ECGF), and platelet-derived growth factor-C (PDGF-C). Minoxidil increased extracellular signal-regulated kinases 1/2 (ERK1/2) phosphorylation, and concomitant upregulation of PD-ECGF and PDGF-C mRNA levels were attenuated by an ERK inhibitor. Subcutaneous injection of CXCL1, PD-ECGF, or PDGF-C enhanced anagen induction in mice, and both CXCL1 and PDGF-C increased hair length in ex vivo organ culture. Treatment with CXCL1, PD-ECGF, or PDGF-C also increased the proliferation index in DP cells. Finally, topical application of CXCL1, PD-ECGF, or PDGF-C with $\%$ minoxidil enhanced anagen induction when compared to minoxidil alone. Minoxidil stimulates ASC motility and increases paracrine growth factor signaling. Minoxidil-stimulated secretion of growth factors by ASCs may enhance hair growth by promoting DP proliferation. Therefore, minoxidil can be used as an ASC preconditioning agent for hair regeneration.
\end{abstract}

Keywords: minoxidil; adipose-derived stem cells; hair growth; CXCL1; PD-ECGF; PDGF-C

\section{Introduction}

Adipose-derived stem cells (ASCs) have stimulatory effects on dermal papilla (DP) cells to promote hair-growth [1-6]. For example, ASCs secrete multiple growth factors, such as vascular endothelial growth factor (VEGF) and basic fibroblast growth factors (bFGF), which can increase the proliferation of DP cells [1]. Festa et al. showed that adipocyte lineage cells drive hair cycling by contributing to the skin stem cell niche, and suggested that platelet-derived growth factor-A (PDGF-A) expression by immature adipocytes regulates follicular stem cell activity [2]. Grafting of ASC-enriched adipose tissue (i.e., by injection of the stromal vascular fraction of lipoaspirate) has shown promise as 
an alternative approach to treating baldness in men and women [7]. We have identified stimulators that enhance the hair-regenerative potential of ASCs in vivo. For instance, vitamin C and low-dose ultraviolet B (UVB) increased secretion of hair growth-promoting factors by ASCs and induced anagen in animal models $[3,4]$. Of the potential stimulators tested, we found that platelet-derived growth factor-D (PDGF-D) exhibited the strongest effects on ASCs and increased secretion of growth factors via mitogen-activated protein kinase (MAPK) pathways [5]. In addition, CAP-18 for cathelicidin antimicrobial peptide (LL-37) increased the secretion of growth factors and the hair-regenerative efficacy of ASCs via early growth response 1 (ERG1) protein and the MAPK pathway [8].

Minoxidil was first developed to treat male- and female-pattern alopecia. In addition to vasodilation, there is strong evidence that minoxidil directly promotes hair growth via the stimulation of DP and epithelial cells [9-14]. Minoxidil stimulated mouse vibrissae follicles in organ culture and induced proliferation of hair epithelial cells near the follicle base [9]. Further, minoxidil and its derivatives showed cytoprotective activity in vivo and increased prostaglandin E2 (PGE2) production by human DP fibroblasts [11]. In cultured DP cells, minoxidil-induced hair growth was mediated by adenosine receptors [12]. Minoxidil also promoted the survival of human DP cells by activating both the ERK and protein kinase B (Akt) pathways, and prevented apoptotic cell death by increasing the ratio of Bcl-2/Bax [15]. Moreover, minoxidil activated the $\beta$-catenin pathway in human DP cells, suggesting a possible mechanism for its anagen prolongation effect [13]. Minoxidil suppressed androgen receptor (AR)-mediated functions by decreasing AR transcriptional activity in reporter assays and reducing expression of AR targets at the protein level [16]. Otomo summarized the primary mechanisms of minoxidil action as (a) induction of growth factors in DP cells, such as VEGF, hepatocyte growth factor (HGF), and insulin-like growth factor-1 (IGF-1); (b) inhibition of TGF- $\beta$-induced apoptosis of hair matrix cells; and, (c) increase of blood flow by dilating hair follicle arteries [14]. However, there is little evidence that minoxidil can indirectly promote hair growth via ASCs, even though ASCs contribute to the stem cell niche for hair follicles and exert stimulatory effects on hair cycle progression. Therefore, in the present study, we examined possible indirect hair growth-promoting effects of minoxidil via ASCs. Specifically, we investigated whether minoxidil stimulates growth factor secretion by ASCs to enhance follicular cell activity and hair growth.

\section{Results}

\subsection{Minoxidil-Pretreated Adipose-Derived Stem Cells (ASCs) Promote Hair Growth In Vivo}

ASCs are known to stimulate hair growth [1-6]. We found that injection of naïve (untreated) human ASCs increased telogen-to-anagen induction in mice only slightly following subcutaneous injection, while ASCs pretreated with minoxidil induced robust hair growth (Figure 1A,B). To examine the effect of ASCs pretreated with minoxidil on hair follicle, we performed hematoxylin and eosin (HE) staining and immunofluorescence staining for Ki67, which is a proliferating cell marker in DP. The skin section of $\mathrm{ASC}^{\mathrm{MXD}}$-treated mice showed higher number of mature hair follicle compared to vehicleor ASC ${ }^{\mathrm{Ctrl}}$-treated mice (Figure 1C). In addition, most hair follicles of $\mathrm{ASC}^{\mathrm{MXD}}$-treated mice showed DP with Ki $67^{+}$cells contrary to vehicle- or $\mathrm{ASC}^{\mathrm{Ctrl}}$-treated mice (Figure 1D). This result suggests that minoxidil can promote telogen to anagen induction, thereby promoting hair growth. 

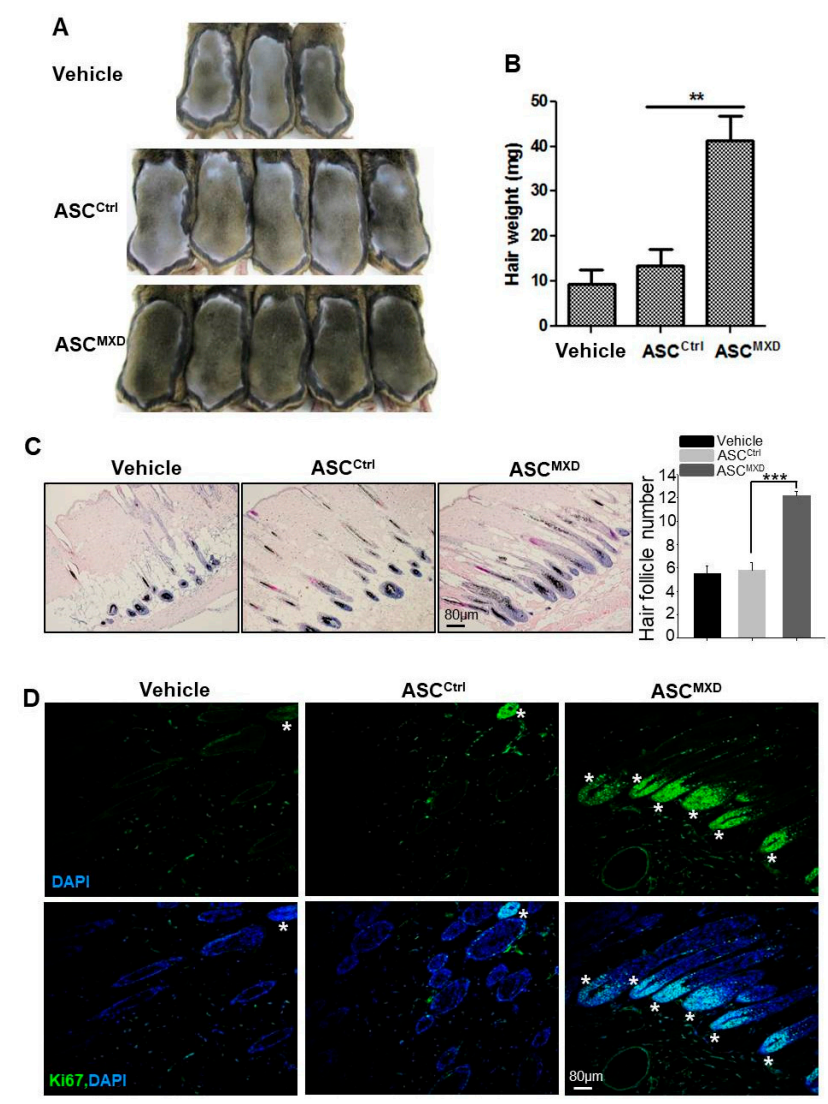

Figure 1. Adipose-derived stem cells (ASCs) pretreated with minoxidil promote hair growth in vivo. Minoxidil-treated ASCs or untreated ASCs were injected into the dorsal skin of shaved mice. Photograph was taken (A), and hair weight measured (B) 14 days later; (C) Skin section was analyzed by HE staining and the number of mature hair follicle was measured; (D) The hair follicle with $\mathrm{Ki}^{+}$cells in DP was shown by immunostaining. Asterisks indicate hair follicles with $\mathrm{Ki} 67^{+} \mathrm{DP}$ cells. ${ }^{* *} p<0.01,{ }^{* * *} p<0.001 . n=7$ or 8 mice per group. All error bars indicate SEM.

\subsection{Minoxidil Can Induce Migration of ASCS}

To examine whether minoxidil affects ASC proliferation, we determined the live cell number over two days and seven days of minoxidil treatment. Minoxidil had no effect on ASC proliferation under either condition, even at the highest dose (Figure 2A,B). To explore whether minoxidil affects ASC migration, we conducted scratch and transwell migration assays. Minoxidil at 20 and $50 \mu \mathrm{M}$ dose-dependently increased ASC migration into both the scratch wound assay (Figure 2C) and transwell migration assay (Figure 2D). To determine whether the effect of minoxidil on specific to the ASCs, we examined the effect of minoxidil on growth and migration of dermal fibroblast cells. Minoxidil did not induce cell growth either migration (Figure S1). Initially, topically applied minoxidil was believed to stimulate hair growth by indirect actions, such as vasodilatation and increased blood flow to the DP $[10,14]$. Therefore, we examined whether minoxidil affects blood vessel formation by ASCs using an in vitro tube formation assay. Indeed, minoxidil dose-dependently increased the number of nascent tubes after 12-16 h (Figure 2E) and the expression level of endothelial cell markers including tyrosine kinase with immunoglobulin-like and EGF-like domains 1 (TIE1), vascular endothelial growth factor receptor 1 (VEGFR1), VEGFR2 and endothelin receptor type B (EDNRB) (Figure 2F). Collectively, these results suggest that minoxidil may promote hair growth by enhancing ASC migration and ASC-dependent angiogenesis. 
A

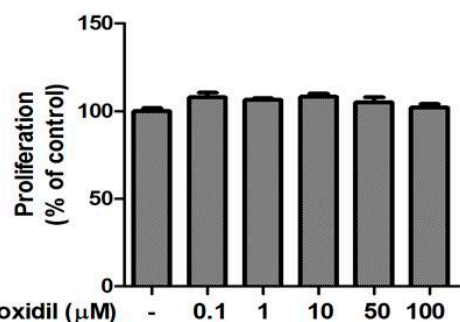

B

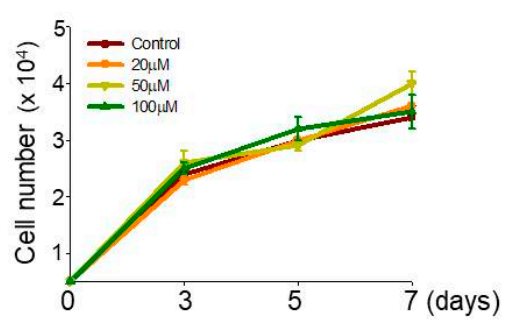

C

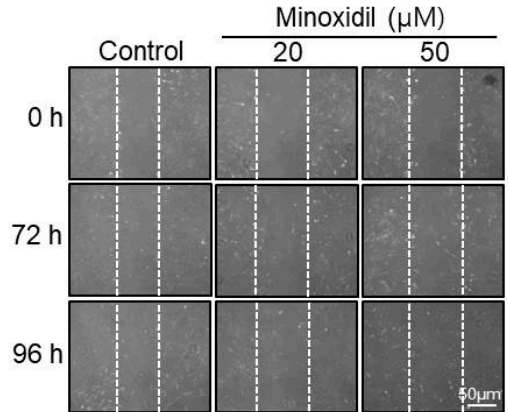

D

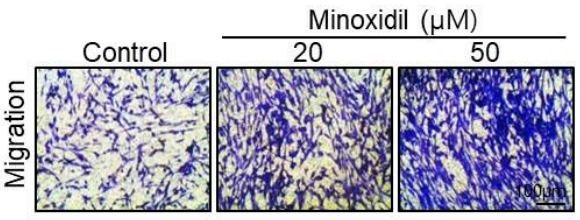

$\mathbf{E}$

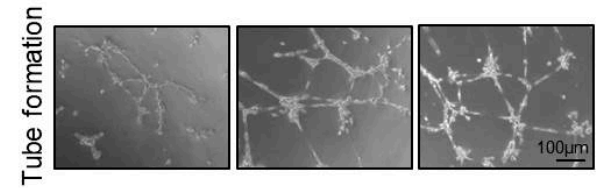

$\mathbf{F}$

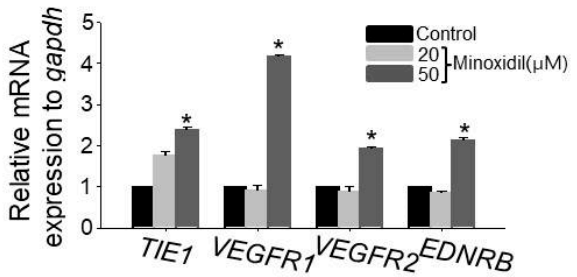

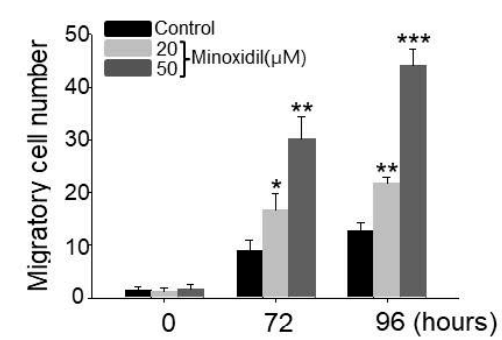
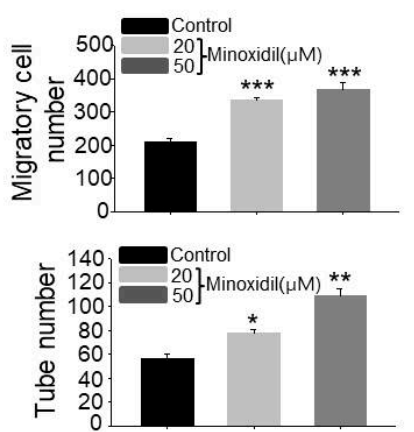

Figure 2. Minoxidil promotes ASC migration and tube formation but not proliferation. (A,B) No effect of minoxidil on proliferation of ASCs. (C,D) Minoxidil enhances ASC migration as evidenced by both scratch migration assay (C) and transwell migration assay (D); (E) Tube formation assay showing enhanced formation by minoxidil-treated ASCs. (F) Relative mRNA expression levels of endothelial cell markers including TIE1, VEGFR1, VEGFR2, and EDNRB genes were increased in tubes from minoxidil-treated ASCs. ${ }^{*} p<0.05,{ }^{* *} p<0.01,{ }^{* * *} p<0.001$. Three independent experiments were conducted per data point. All error bars indicate SEM.

\subsection{CXCL1, ECGF, and PDGF-C Induce Hair Growth}

It has been reported that growth factors secreted by ASCs, such as VEGF, fibroblast growth factor-1 (FGF1), bFGF, and PDGF-A, regulate hair follicular stem cell activity and induce the anagen phase of the hair cycle in vivo [14]. We speculated that minoxidil may promote hair growth indirectly by enhancing growth factor release from ASCs. To explore this possibility, we compared expression patterns between untreated naïve and minoxidil-treated ASCs by qPCR array. Minoxidil upregulated the expression of PD-ECGF over six-fold when compared to untreated ASCs (Figure S2), and this result was confirmed (Figure 3A). Moreover, minoxidil also upregulated the expression levels of CXCL1 and PDGF-C (Figure 3A). 


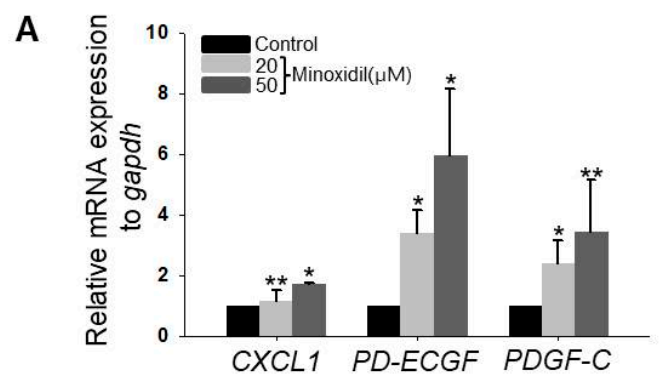

B
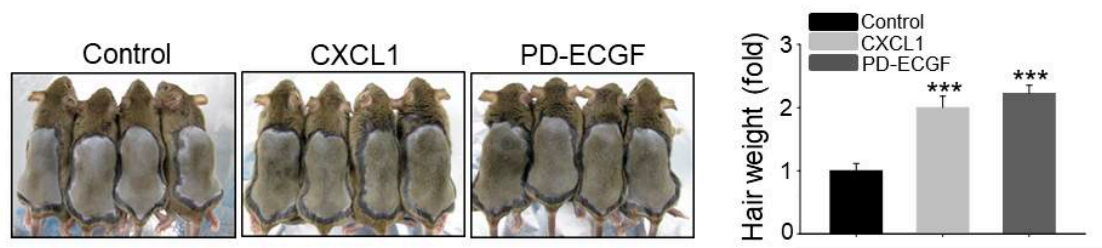

C
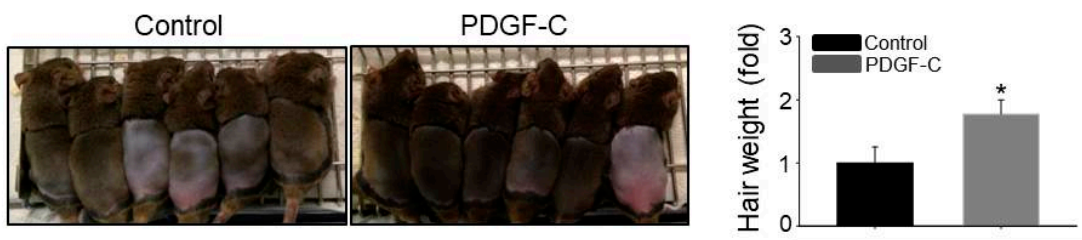

D

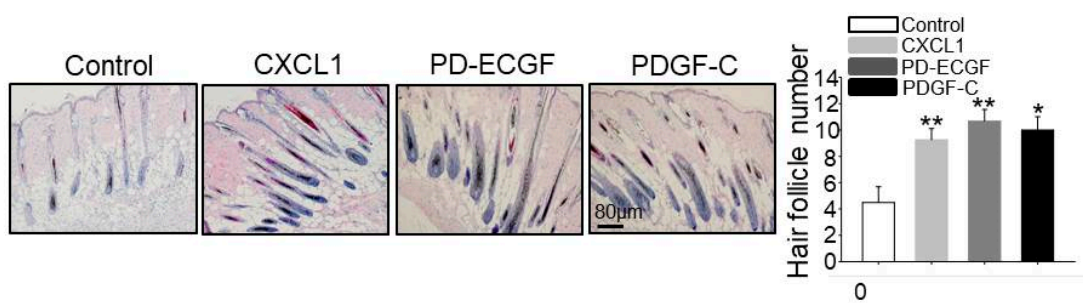

E
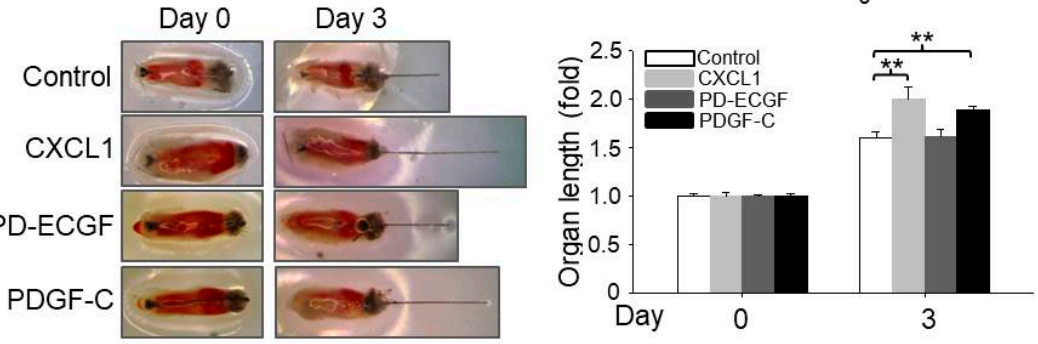

Figure 3. Minoxidil upregulates expression of hair-growth promoting factors CXCL1, PD-ECGF, and PDGF-C in ASCs. (A) Relative mRNA expression levels of growth factors including CXCL1, $P D$-ECGF, and PDGF-C were measured in minoxidil-treated ASCs; (B-D) Human recombinant CXCL1, PD-ECGF, and PDGF-C proteins each facilitated hair growth and increased the number of mature hair follicle after injection into the dorsal skin of shaved mice as revealed by hair weight at 2 weeks post-injection. $n=6-8$ mice per group. ${ }^{*} p<0.05,{ }^{* *} p<0.01,{ }^{* * *} p<0.001$. (E) CXCL1, PD-ECGF, and PDGF-C proteins each facilitate mouse vibrissal hair follicle growth ex vivo. ${ }^{* *} p<0.01$. $n=10$ samples per treatment group. All error bars indicate SEM.

To investigate whether CXCL1, PD-ECGF, and PDGF-C can induce the anagen phase of the hair cycle in vivo, we injected recombinant human CXCL1, PD-ECGF, or PDGF-C protein into the subcutaneous dermis of shaved mice. All three factors significantly induced the anagen phase of the hair cycle in vivo (Figure 3B,C) and increased the number of mature hair follicle (Figure 3D), suggesting that minoxidil may induce anagen by triggering CXCL1, PD-ECGF, or PDGF-C release from ASCs. Moreover, treatment with CXCL1 or PDGF-C, but not PD-ECGF, also increased the length 
of isolated mouse vibrissal hair follicles in organ culture (Figure 3E). These results strongly suggest that minoxidil promotes hair growth through growth factor release from ASCs.

\subsection{Minoxidil Regulates Expression of PD-ECGF and PDGF-C in ASCs through the ERK Pathway}

It has been reported that the MAPK pathway regulates expression of growth factors in ASCs, including vascular endothelial growth factor A (VEGFA) and FGF1 [5,8]. Therefore, we examined whether minoxidil regulates the expression of CXCL1, PD-ECGF, or PDGF-C through the MAPK pathway. Indeed, minoxidil dose-dependently upregulated phospho-ERK expression, a response suppressed by the specific mitogen-activated protein kinase kinase (MEK) inhibitor PD98059 (Figure 4A). Further, PD98059 reversed minoxidil-induced upregulation of PD-ECGF and PDGF-C in ASCs (Figure 4B). Alternatively, minoxidil-induced upregulation of CXCL1 was not affected by PD98059 (Figure 4B), suggesting that minoxidil upregulates growth factor expression in ASCs through multiple pathways, including the MAPK pathway.

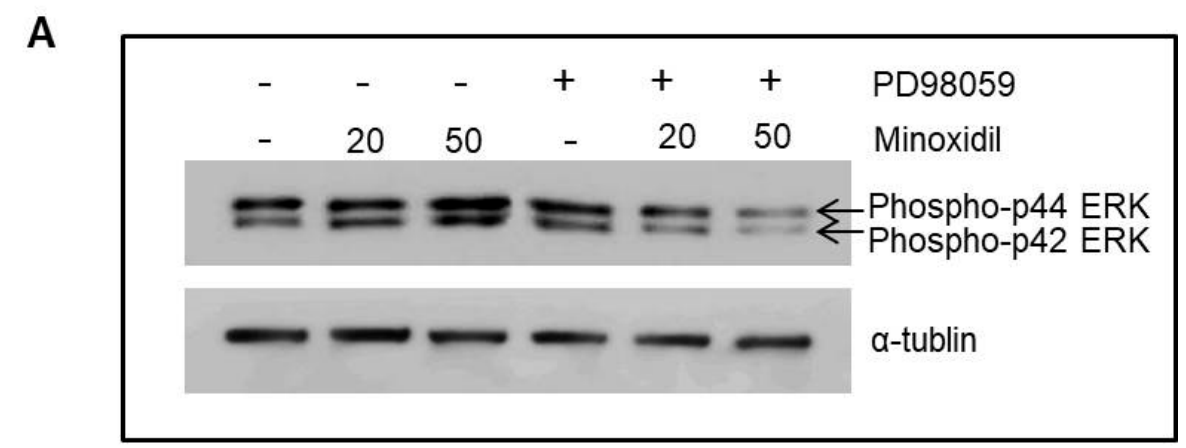

\section{B}

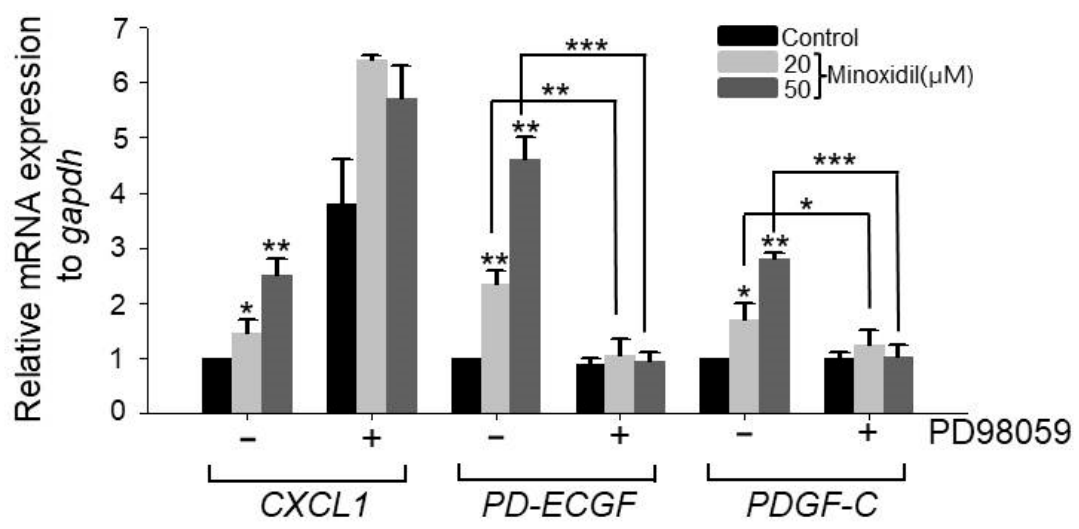

Figure 4. Minoxidil upregulates expression of $P D-E C G F$ and $P D G F-C$ through the ERK pathway in ASCs. (A) The MEK inhibitor PD98059 reversed minoxidil-induced ERK phosphorylation; (B) PD98059 also suppressed minoxidil-induced upregulation of PD-ECGF and PDGF-C expression by ASCs. Three independent experiments were carried out per data point. ${ }^{*} p<0.05,{ }^{* *} p<0.01,{ }^{* * *} p<0.001$. All the error bars indicate SEM.

\subsection{ASC Growth Factors CXCL1, PD-ECGF, and PDGF-C Induce DP Cell Proliferation}

It has been reported that minoxidil stimulates the growth of human hairs by prolonging anagen through proliferative and anti-apoptotic effects on DP cells $[13,15]$. We therefore directly examined whether CXCL1, PD-ECGF, or PDGF-C increase DP cell proliferation, and indeed, all three factors when 
applied separately dose-dependently increased cultured DP cell proliferation (Figure 5A). To confirm this observation is specific to DP cells, we examined whether CXCL1, PD-ECGF, or PDGF-C increase the proliferation of human dermal fibroblast cells. All three proteins did not induce the proliferation of fibroblast contrary to increase in DP cells (Figure S3). Further, all three factors enhanced the number of DP cells in S-phase as evidenced by 5-bromo-2'-deoxyuridine (BrdU) labeling. Moreover, treatment of CXCL1, PD-ECGF, or PDGF-C in DP cells also dose-dependently increased the percentage of BrdU ${ }^{+}$ cells, which is proliferation index (Figure 5B,C). These results further suggest that minoxidil may enhance DP proliferation indirectly by inducing release of growth factors, such as CXCL1, PD-ECGF, and PDGF-C from ASC.
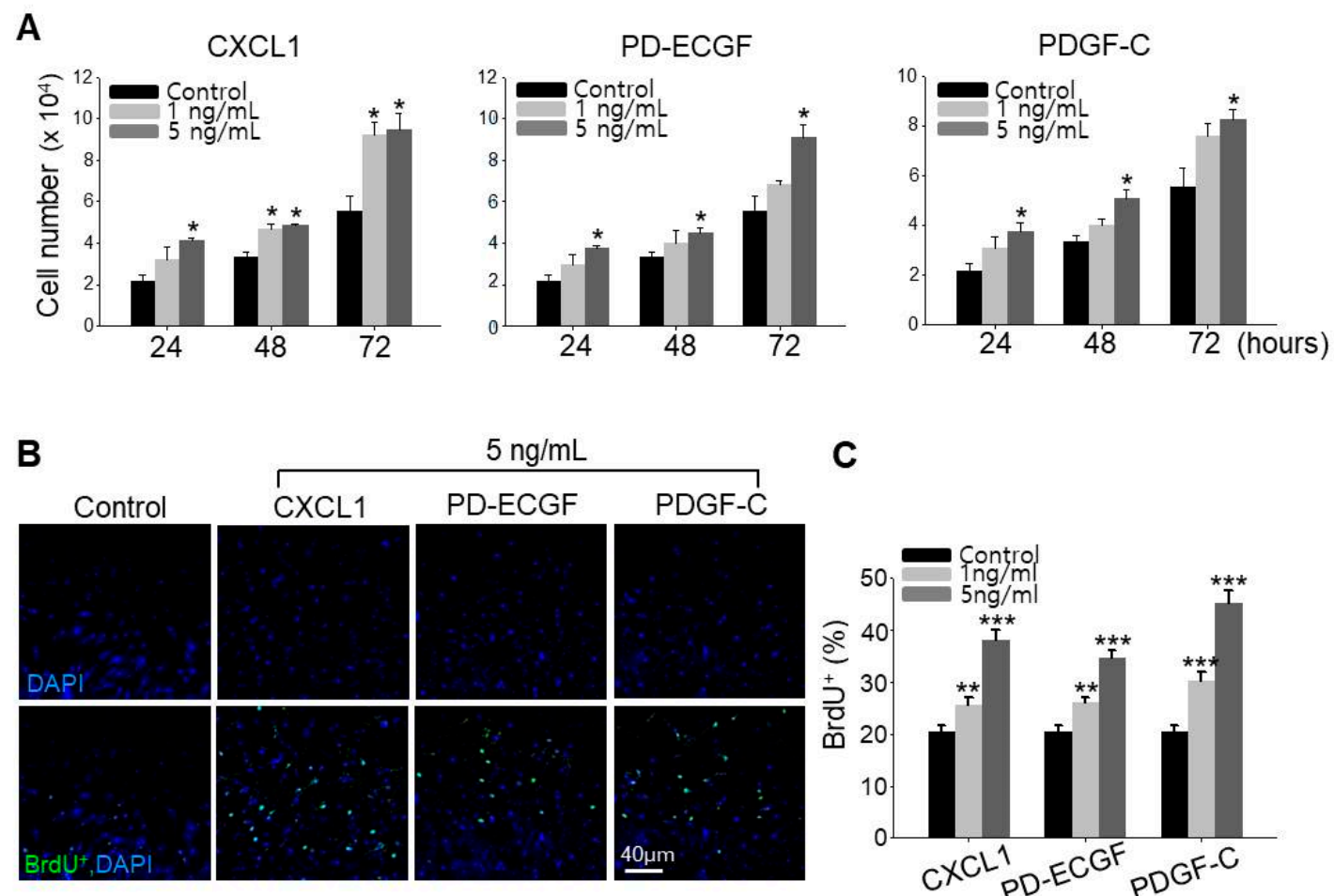

$5 \mathrm{ng} / \mathrm{mL}$

C
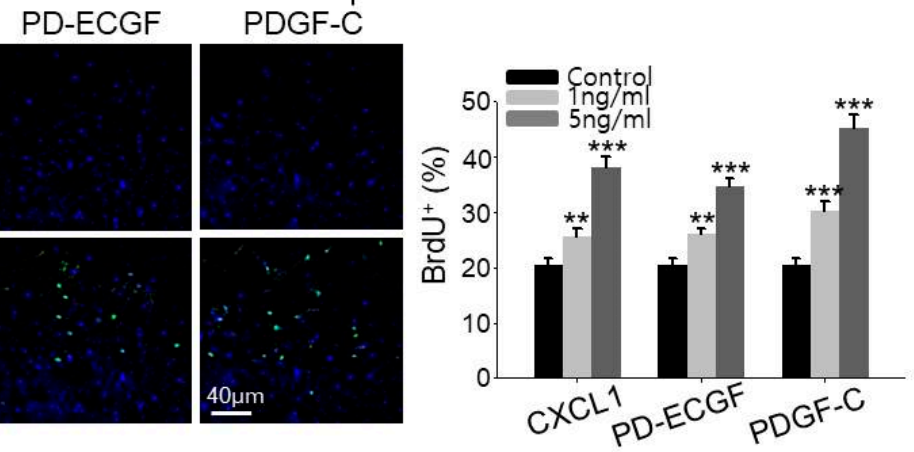

Figure 5. CXCL1, PD-ECGF, and PDGF-C induce proliferation of DP cells. (A) Cell growth was measured after treatment of CXCL1, PD-ECGF, or PDGF-C protein in DP cells for 3 days. Three independent experiments were conducted. ${ }^{*} p<0.05$. (B,C) Proliferation index $\left(\%\right.$ of BrdU ${ }^{+}$ cells; green) after treatment of CXCL1, PD-ECGF, or PDGF-C protein in DP cells. Three independent experiments were carried out per data point. ${ }^{* *} p<0.01,{ }^{* * *} p<0.001$. All error bars indicate SEM.

\subsection{Application of CXCL1, PD-ECGF, or PDGF-C Acts Synergistically with Minoxidil to Induce Hair Growth}

Our results suggest that upregulation of CXCL1, PD-ECGF, or PDGF-C by minoxidil stimulates DP cell proliferation, resulting in hair growth. Minoxidil has been widely used to treat androgenetic alopecia. Therefore, to investigate whether application of CXCL1, PD-ECGF, or PDGF-C acts synergistically with $2 \%$ minoxidil to induce the anagen phase of the hair cycle, we compared hair growth on the dorsal skin among shaved mice that were treated with $2 \%$ minoxidil alone or minoxidil plus either CXCL1, PD-ECGF, or PDGF-C for 14 days. Co-administration of each protein increased hair weight when compared to $2 \%$ minoxidil alone (Figure $6 \mathrm{~A}, \mathrm{~B}$ ), suggesting that the addition of these proteins may enhance the efficacy of minoxidil. 
A
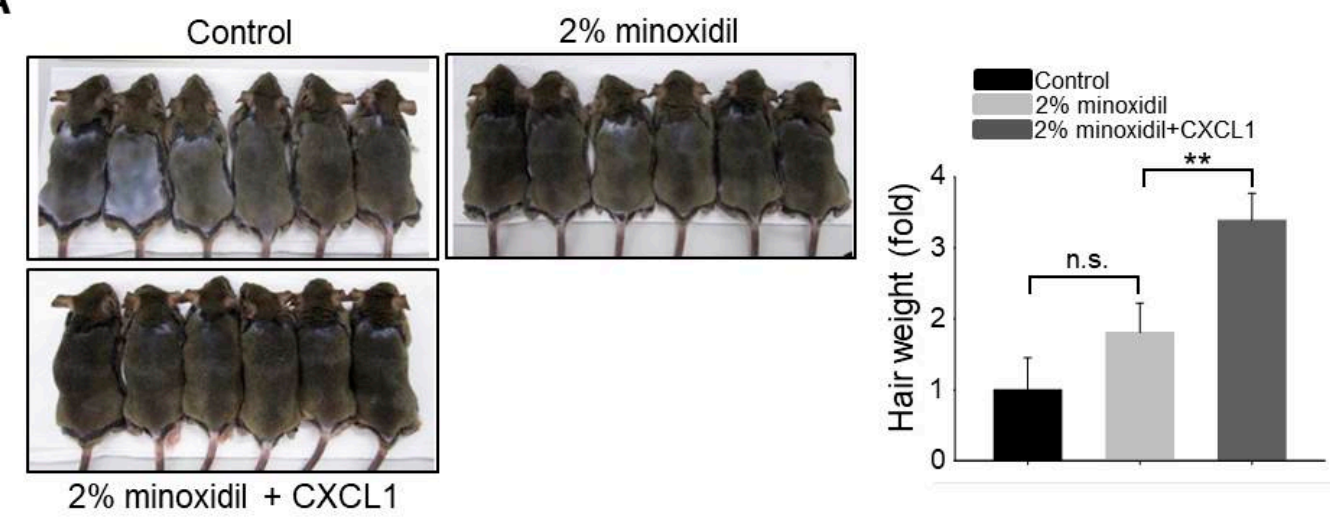

B

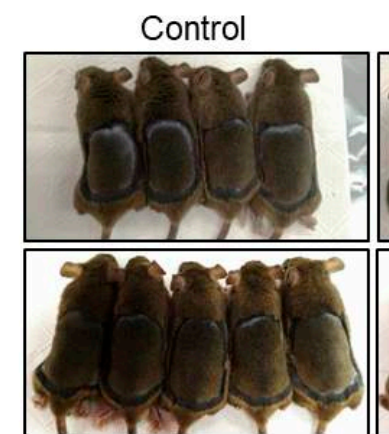

$2 \%$ minoxidil + PD-ECGF

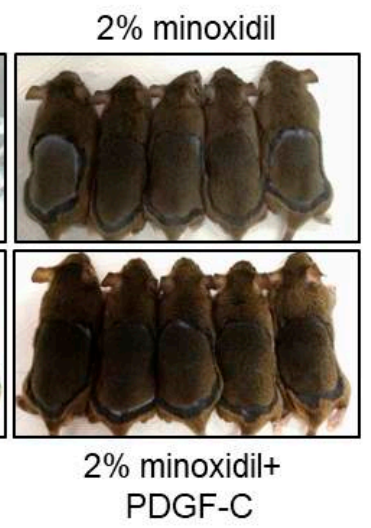

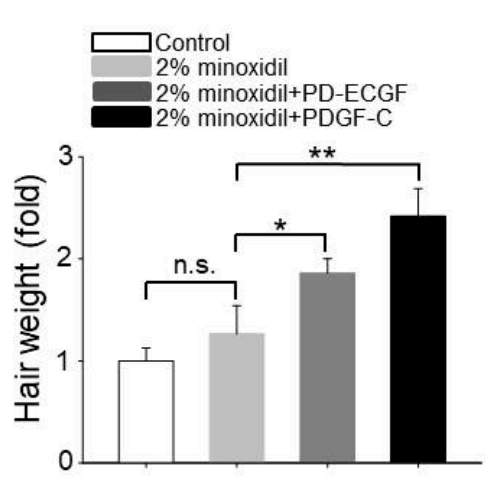

Figure 6. Application of CXCL1, PD-ECGF, or PDGF-C enhances the hair growth-promoting effect of minoxidil (2\%) when co-applied on the dorsal skin of shaved mice. (A) Photograph was taken and hair weight was measured 14 days later for CXCL1 application. ${ }^{* *} p<0.01 . n=6$ mice per group; (B) Photograph was taken and hair weight was measured 14 days later for PD-ECGF or PDGF-C application. ${ }^{*} p<0.05,{ }^{* *} p<0.01$. n.s. indicates not significant. $n=5$ or 6 mice per group. All of the error bars indicate SEM.

\section{Discussion}

Minoxidil promotes hair growth directly by stimulating DP and epithelial cells, but previous studies provided little or no evidence for indirect hair growth-promoting effects through the stimulation of ASCs. Therefore, we investigated whether minoxidil stimulates ASCs and enhances hair growth through growth factor release. We first demonstrated that subcutaneous injection of minoxidil-treated ASCs accelerated telogen-to-anagen transition in $\mathrm{C} 3 \mathrm{H} / \mathrm{HeJ}$ mice and increased hair weight after two weeks. Although minoxidil did not alter the proliferation of ASCs, it did increase migration, tube formation, and secretion of growth factors, notably CXCL1, PD-ECGF, or PDGF-C (the later two through ERK activation). Further, each of these growth factors enhanced DP cell proliferation in vitro and anagen induction in mice, while CXCL1 and PDGF-C also increased the length of isolated mouse vibrissal hair follicles in organ culture. Moreover, these hair growth-promoting effects were synergistic with $2 \%$ minoxidil. Collectively, these results indicate that minoxidil stimulates hair growth in part through stimulation of CXCL1, PD-ECGF, or PDGF-C release from ASCs (Figure 7). 


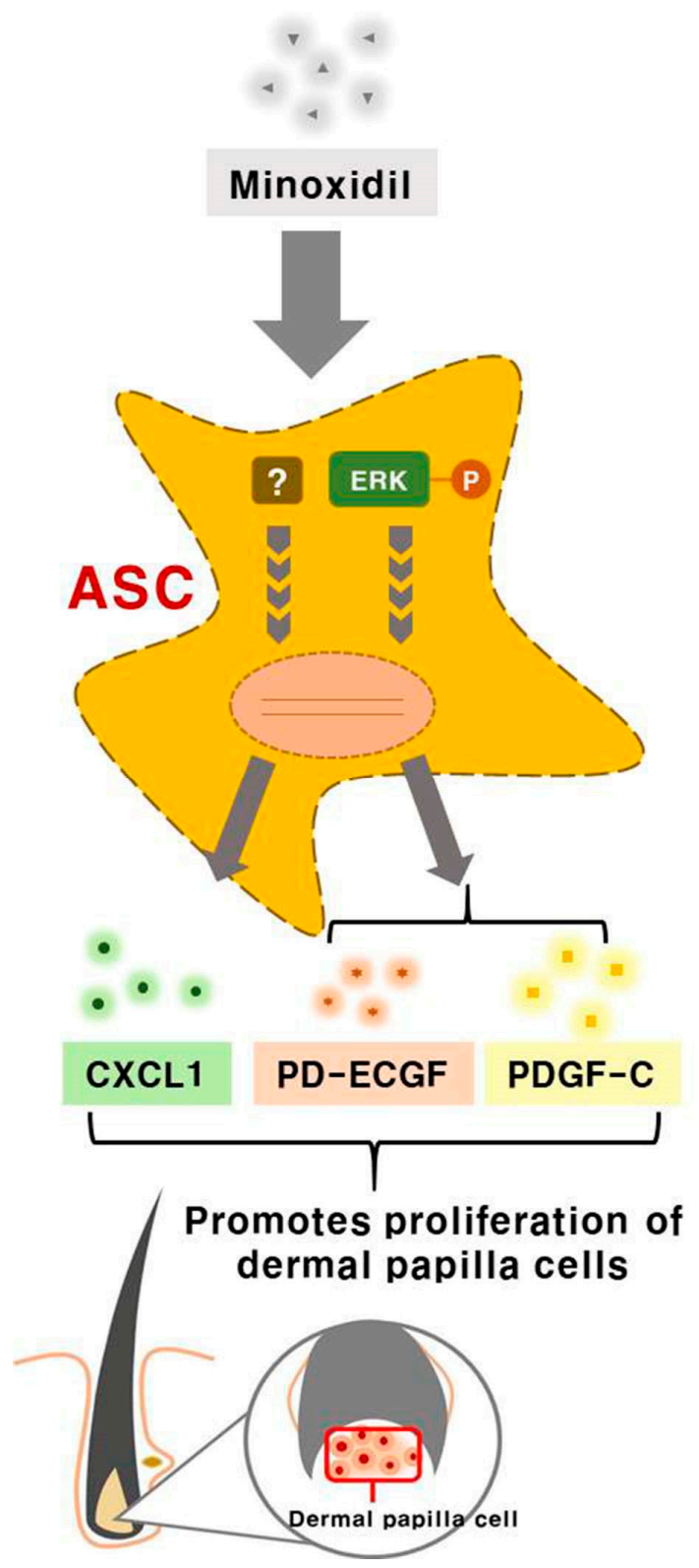

Figure 7. Minoxidil promotes the proliferation of DP cells and hair growth through stimulation of growth factor release from adipose-derived stem cells. Minoxidil stimulates the release of growth factors including PD-ECGF, PDGF-C, and CXCL1 from ASCs via ERK and the other pathway, respectively, thereby promoting of DP cells proliferation and hair growth.

Chemokine (C-X-C motif) ligand 1 is a secreted growth factor that signals through the G-protein coupled CXC receptor 2, while CXCL1 is known to act as a potent chemoattractant for neutrophils during inflammation [17-19]. However, it has not been linked to hair regeneration. PD-ECGF promotes angiogenesis in vivo and stimulates the in vitro growth of multiple endothelial cell types [20-22]. It has a highly restricted target cell specificity, acting only on endothelial cells. Of interest, $P D$-ECGF was one of the top three genes that were upregulated in ASCs by minoxidil according to qPCR arrays. Moreover, our work in vitro and in vivo revealed that minoxidil-induced PD-ECGF release from ASCs, resulting in DP proliferation and hair growth. On the contrary, it has been well-known that PDGF signaling in the dermis and in dermal condensates is dispensable for hair follicle induction and formation [23]. For example, PDGF-A secreted from ASCs regulates follicular stem cell activity [2]. 
PDGF-A and -B are involved in the induction and maintenance of the anagen phase in the mouse hair cycle [24]. Our work revealed that PDGF-C also functions in hair growth by upregulating DP proliferation. Although these results might be expected because many growth factors are already known to promote hair cycling, upregulation by minoxidil is meaningful for further usability of this agent to treat androgenetic alopecia. Indeed, the co-application of PDGF-C, CXCL1, or PD-ECGF with $2 \%$ minoxidil synergistically increased hair growth (Figure 6).

It has been reported that PDGF-D treatment induces growth factor secretion from ASCs via phospho-activation of ERK [5,8]. Meldrum's group also reported that preconditioning (i.e., by hypoxia or transforming growth factor) induced the secretion of numerous growth factors from mesenchymal stem cells through the MAPK pathways [25-28]. Similarly, our work revealed that minoxidil induced PD-ECGF and PDGF-C release from ASCs via ERK phosphorylation (Figure 4). Therefore, it is reasonable to assume that growth factor secretion by ASCs is primarily regulated by the MAPK pathway. However, it appears that CXCL1 is induced via an ERK-independent pathway, suggesting that minoxidil may activate multiple signaling pathways in ASCs. Further investigation is required to identify the pathway mediating minoxidil-induced CXCL1 release.

Minoxidil reportedly increased the proliferation of DP cells in vitro. For example, minoxidil promoted the survival of human DP cells by activating both ERK and Akt pathways, and prevented cell death by increasing the ratio of Bcl-2/Bax [15]. In addition, minoxidil plus all-trans retinoic acid (ATRA) additively promoted hair growth in human hair follicle culture [29]. A combination of minoxidil with ATRA elevated phosphorylated ERK, phosphorylated Akt in DP cells and keratinocytes [29]. However, minoxidil did not increase the proliferation in ASCs, which indicates that proliferative effect of minoxidil is cell type dependent.

ACSs are considered an important component of other stem cell niches. Indeed, it has been shown that adipocyte lineage cells are part of the skin stem cell niche that drives hair cycling, and it was suggested that PDGF-A expression by immature adipocytes is a key regulator of follicular stem cell activity [2]. Although we did not investigate the effects of minoxidil or ASC-released growth factors on DP stem cells, ASC-released growth factors (specifically CXCL1, PD-ECGF and PDGF-C) induced DP cell proliferation, consistent with a pivotal role in the regulation of DP stem cells. To determine whether the effect of minoxidil on specific to the ASCs, minoxidil didn't induce cell growth either migration of dermal fibroblast cells (Figure S1).

We previously reported that preconditioned ASCs by growth factor, such as PDGF-D, enhance hair generative potential in tellogen to anagen induction model [5]. The purpose of this model is to induce anagen phase more quickly after we injected ASCs by paracrine effect. Anagen hair induction was not limited to the injection site. Instead, darkening of the skin or hair appeared across all areas of the back. We showed increased hair weight in $\mathrm{ASC}^{\mathrm{MXD}}$-treated mice, which indicates the increase of hair length as well as hair number. The measurement of hair weight as an evaluation for hair growth is not enough to conclude increased telogen-to-anagen induction. Therefore, we examined hair follicle and proliferating DP cells in sectioned skin. The skin section of $\mathrm{ASC}^{\mathrm{MXD}}$-treated mice showed higher number of mature hair follicle compared to vehicle- or ASC ${ }^{\text {Ctrl }}$-treated mice (Figure 1C). In addition, most hair follicles of $\mathrm{ASC}^{\mathrm{MXD}}$-treated mice showed DP with $\mathrm{Ki}^{+} 7^{+}$cells contrary to vehicle- or $\mathrm{ASC}^{\mathrm{Ctrl}}$-treated mice (Figure 1D). This result suggests that minoxidil can promote telogen to anagen induction, thereby promoting hair growth. Although the measurement of hair weight is not enough to examine the growth of hair follicle and DP proliferation, this method is easy to monitor hair growth quickly.

In summary, subcutaneous injection of minoxidil-treated ASCs accelerated the telogen-to-anagen transition in mice, and direct minoxidil treatment increased migration, tube formation, and growth factor secretion by ASCs. The most strongly upregulated growth factors, CXCL1, PD-ECGF and PDGF-C, individually enhanced anagen induction in mice, while CXCL1 and PDGF-C also increased the length of isolated mouse vibrissal hair follicles in organ culture. Therefore, minoxidil can be used as a novel ASC preconditioning agent for hair regeneration. 


\section{Materials and Methods}

\subsection{Cell Culture}

Human adipocyte-derived stem cells (ASCs) were isolated via liposuction of subcutaneous fat as described previously [30,31]. Briefly, fat was washed with phosphate-buffered saline (PBS), added $0.075 \%$ collagenase and incubated for $45 \mathrm{~min}$ at $37{ }^{\circ} \mathrm{C}$ with gently shaking. The pellet after centrifugation was filtered with $100 \mu \mathrm{m}$ nylon mesh, gathered again after centrifugation. Then, cells were cultured with essential medium, including $\alpha$-minimum essential media (MEM) (Hyclone, Logan, UT, USA), 10\% fetal bovine serum (Gibco, Carlsbad, CA, USA) and 1\% anti-antibiotics (Gibco) until passage 3. Then, medium was changed to minimum essential medium including $\alpha$-MEM, $10 \%$ fetal bovine serum, and 1\% penicillin/streptomycin (Gibco) at four passages. ASCs were used at passages 5-7 for all of the experiments. Characterization of ASCs was performed using flow cytometry. ASCs were positive for CD44, CD73, CD90, CD105, human leukocyte antigen-I (HLA-I), and podocalyxin (PODXL), but were negative for hematopoietic markers such as CD34 and CD45 [32,33]. Multipotent differentiation potential was examined as described previously [34,35], and ASCs could be differentiated into adipocytes, osteocytes, and chondrocytes. We purchased human DP cells from PromoCell (\#C-12071, PromoCell, Logan, Heidelberg, Germany). Human DP cells were cultured in Follicle DP Cell Medium (C-26505; PromoCell) with SupplementMix (C-39625; PromoCell) and $0.1 \%$ anti-antibiotics (Gibco). DP cells were used at passages 3, 4 . We also purchased human dermal fibroblast cells from PromoCell (\#C-12302). Dermal fibroblasts were cultured in dulbecco modified eagle medium (DMEM)/high glucose (Hyclone) with $10 \%$ fetal bovine serum and $1 \%$ penicillin/streptomycin, and were used at passages 8-10. All of the cells were maintained at $37{ }^{\circ} \mathrm{C}$ in a humidified $5 \% \mathrm{CO}_{2}$ incubator (Theremo Fisher scientific, Waltham, MA, USA).

\subsection{Cell Growth Assay}

For measuring the effects of minoxidil on ASC proliferation, cells were seeded in 6-well plates at $1 \times 10^{4}$ cells/well, treated with minoxidil (0.1-100 $\left.\mu \mathrm{M}\right)$, and incubated for two days in an InCu-saFe $\mathrm{CO}_{2}$ incubator (Panasonic, Kadoma, Osaka, Japan). Live cell number was determined using the IncuCyte zoom2014A live cell analysis system (Panasonic, Kadoma, Osaka, Japan) [5]. Alternatively, ASCs were seeded in 12-well plates at 5000 cells/well, treated with minoxidil (20, 50, or $100 \mu \mathrm{M})$, and incubated for seven days. Cells were then trypsinized, stained with Trypan Blue (Sigma-Aldrich, St. Louis, MO, USA), and counted each day using a hemocytometer hemocytometer. To assess the effects of growth factors secreted by ASCs on DP cell proliferation, $1 \times 10^{4}$ DP cells were seeded in 12-well plates and treated with the indicated synthesized peptides for up to three days. Cells were trypsinized and stained with Trypan Blue. Viable cell number was counted each day using a hemocytometer.

\subsection{Scratch Migration Assay}

Cells were seeded into 6-well plates and cultured to confluence. A sterile $1 \mathrm{~mL}$ pipette tip was used to scratch the cell monolayer. Cultures were then washed with PBS to removed deplated cells and cultured with minoxidil in serum-free medium for four days. Migration of cells into the scratched area (wound closure) was visualized using a ZEISS Observer D1 microscope (Carl ZEISS, Oberkochen, Germany). Multiple images were acquired per well and average cell counts within the wound monitored over 4 days.

\subsection{Cell Migration Assay Using Transwell}

ASCs $\left(1 \times 10^{4} /\right.$ well $)$ were suspended in serum-free medium and seeded on the upper side of transwell membrane plates (BD falcon, BD Biosciences, San Jose, CA, USA). After 2 h, minoxidil in serum-free medium were introduced into the lower chambers. Cultures were incubated for two days to allow transwell migration. Inserts were then removed and the upper surface was cleaned 
of non-migrating cells using cotton swabs and washed with PBS. The inserts were stained with $0.1 \%$ formalin $/ 10 \%$ crystal-violet solution (Sigma-Aldrich) and cell number analyzed under a ZEISS Observer D1 microscope. Multiple images (15-20) per insert were acquired, and average cell counts were calculated.

\subsection{Tube Formation Assay Using Matrigel}

Twelve-well plates were coated with matrigel (BD matrigel matrix, BD Biosciences) and were dried for $2 \mathrm{~h}$ at $37^{\circ} \mathrm{C}$. ASCs in endothelial cell basal medium-2 (EBM-2, LONZA, Walkersville, MD, USA) plus minoxidil were plated on matrigel-coated wells and incubated for $12-16 \mathrm{~h}$ at $37^{\circ} \mathrm{C}$. The number of tubes was analyzed under a ZEISS Observer D1 microscope [3], and the expression level of endothelial cell markers was analyzed by qPCR.

\subsection{RNA Extraction and Quantitative RT-PCR}

Total RNA was extracted from ASCs using Trizol reagent (Invitrogen, Carlsbad, NY, USA) and subjected to cDNA synthesis using oligodT and the HelixCript ${ }^{\mathrm{TM}}$ Thermo Reverse Transcription System (Nanohelix, Madison, WI, USA), according to the manufacturer's instructions. BrightGreen qPCR master mix-ROX (ABM, New York, NY, USA) was used for PCR reactions.

\subsection{Western Blot}

For western blot of phospho-ERK, minoxidil and PD98059 (Sigma-Aldrich) was treated for $30 \mathrm{~min}-1 \mathrm{~h}$. Cells were lysed with protein extraction solution (PRO-PREP ${ }^{\mathrm{TM}}$; iNtRON, Seoul, Korea) containing phosphatase inhibitor $\left(\mathrm{Na}_{3} \mathrm{VO}_{4}\right.$; Roche, Pleasanton, CA, USA). Western blot analysis was performed as described previously [8] using the following primary antibodies: rabbit anti-p42/44 (1:1500; Cell Signaling Technology, Danvers, MA, USA), mouse anti-phospho-p42/44 (1:1500; Cell Signaling Technology), and mouse anti- $\alpha$-tubulin (1:2000; Santa Cruz Biotechnology, Dallas, TX, USA). Western blot images were obtained using ImageQuant LAS 4000 (GE Healthcare Life Science, Pittsburgh, PA, USA).

\subsection{Animal Experiment}

Mice were maintained and anesthetized according to a protocol that was approved by the US Pharmacopoeia and the Institutional Animal Care and Use Committee of Yonsei University (IACUC120002, 17 July 2015). The dorsal area of 6.5-week-old C3H/HeN mice in the telogen stage of the hair cycle was shaved with a clipper and electric shaver, with special care taken to avoid damaging the bare skin. Naïve ASCs or minoxidil-treated ASCs were only once injected into the dorsal skin of shaved mice. The growth factors $(100 \mathrm{ng} / \mathrm{mL}$ per one day) were injected into the dorsal skin of shaved mice every day for 12 days. CXCL1 (R and D Systems, Minneapolis, MN, USA), PD-ECGF (R and D Systems) and PDGF-C (PeproTech, Rocky Hill, NJ, USA) proteins were purchased from each companies. For application with $2 \%$ minoxidil, CXCL1, PD-ECGF or PDGF-C $(1 \mu \mathrm{g} / \mathrm{mL})$ with $2 \%$ minoxidil (Rogaine; Johnson and Johnson Healthcare Products, Skillman, NJ, USA) were applied on the dorsal skin of shaved mice. Any darkening of the skin (indicative of hair cycle induction) was carefully monitored by photography. After 14 days, the dorsal hair was shaved and weighed to estimate growth rate [3,5].

\subsection{Vibrissae Follicle Organ Culture}

For organ culture of vibrissae hair follicle, the vibrissae hair follicle was cut from $\mathrm{C} 3 \mathrm{H} / \mathrm{HeN}$ mice, washed with PBS and cultured in defined medium (Williams E medium supplemented with $2 \mathrm{mM}$ L-glutamine, $10 \mu \mathrm{g} / \mathrm{mL}$ insulin, $10 \mathrm{ng} / \mathrm{mL}$ hydrocortisone, $100 \mathrm{U} / \mathrm{mL}$ penicillin, and $100 \mu \mathrm{g} / \mathrm{mL}$ streptomycin, without serum) including CXCL1 (5 ng), PD-ECGF (20 ng) and PDGF-C (20 ng) for three days. 


\subsection{BrdU Labeling}

For BrdU labeling assay, $4 \times 10^{4}$ cells were seeded in six well plates and incubated for 3 days after adding three proteins. BrdU (Sigma-Aldrich) was added in cell culture media to a final concentration of $200 \mathrm{mM}$ and incubated for $4 \mathrm{~h}$ at $37{ }^{\circ} \mathrm{C}$ with $5 \% \mathrm{CO}_{2}$. The cells were fixed with $4 \%$ paraformaldehyde, incubated with mouse anti-BrdU (1:500) (Abcam, Iowa City, IA, USA) overnight at $4{ }^{\circ} \mathrm{C}$, and then incubated with secondary antibodies, Alexa Fluor 488 goat anti-mouse IgG (Invitrogen), for $1 \mathrm{~h}$ at room temperature with 4,6-diamidino-2-phenylindole (DAPI) (Sigma-Aldrich). Immunofluorescence staining was imaged using ZEISS LSM700 confocal microscope (Carl ZEISS, Oberkochen, Germany). For calculation of the percentage of $\mathrm{BrdU}^{+}$cells, we counted the number of $\mathrm{BrdU}^{+}$cells and $\mathrm{DAPI}^{+}$cells (all cells) in every same size pictures using Adobe photoshop CS6 extended program (Yonsei University, Seoul, Korea), and calculated the percentage of BrdU ${ }^{+}$cells.

\subsection{Hematoxylin/Eosin and Immunofluorescence Staining}

For Hematoxylin and eosin staining, paraffin sections were dewaxed using xylene for $30 \mathrm{~min}$, hydrated in 100\%-, 90\%-, 80\%- and 70\% EtOH and was dipped into Mayer's hematoxylin (Sigma-Aldrich) for $8 \mathrm{~min}$, and then rinsed in water for $1 \mathrm{~min}$. Slide was dipped again into eosin $\mathrm{Y}$ (Sigma-Aldrich) for $80 \mathrm{~s}$, dehydrated with 70\%-, 80\%-, 90\%- and 100\% EtOH, washed with fresh xylene for 30, dried, and mounted with mounting medium. Immunofluorescence staining was performed using standard protocols. Briefly, paraffin sections were dewaxed using xylene for $30 \mathrm{~min}$, hydrated in 100\%-, 90\%-, 80\%- and 70\% EtOH and antigen retrieval was performed by boiling using microwave in antigen retrieval buffer (Dako, Carpinteria, CA, USA) for $2 \mathrm{~min}$. The sections were treated with rabbit Ki67 antibody (1:300) (abcam) overnight at $4{ }^{\circ} \mathrm{C}$, and were then incubated with secondary antibodies, Alexa Fluor 488 goat anti-rabbit IgG (Invitrogen), for $1 \mathrm{~h}$ at room temperature with 4,6-diamidino-2-phenylindole (DAPI) (Sigma-Aldrich). Immunofluorescence staining was imaged using ZEISS LSM700 confocal microscope.

\subsection{Statistical Analysis}

All of the experiments were performed more than three times with independent cultures. Data are presented as mean \pm standard error (SEM). Means were compared by Student's $t$-test. For all statistical tests, a 0.05 level of confidence was accepted as statistically significant.

Supplementary Materials: Supplementary materials can be found at www.mdpi.com/xxx/s1.

Acknowledgments: This study was supported by a grant from the National Research Foundation (NRF-2016R1D1A1B03932050), and a grant from Small and Medium Business Administration (S2371105) by the Korean government. Nahyun Choi was also supported by the National Research Foundation (NRF-2017R1A6A3A11035599) funded by the Korean government.

Author Contributions: Nahyun Choi and Jong-Hyuk Sung designed the experiments; Nahyun Choi developed the methodology and performed the experiments; Nahyun Choi, Jong-Hyuk Sung, Soyoung Shin and Sun U. Song analyzed the data; Nahyun Choi and Jong-Hyuk Sung wrote the paper.

Conflicts of Interest: The authors declare no conflict of interest.

\section{Abbreviations}

ASC Adipose-derived stem cell

CXCL1 chemokine (C-X-C motif) ligand 1

PD-ECGF platelet-derived endothelial cell growth factor

PDGF-C platelet-derived growth factor-C 


\section{References}

1. Won, C.H.; Yoo, H.G.; Kwon, O.S.; Sung, M.Y.; Kang, Y.J.; Chung, J.H.; Park, B.S.; Sung, J.H.; Kim, W.S.; Kim, K.H. Hair growth promoting effects of adipose tissue-derived stem cells. J. Dermatol. Sci. 2010, 57, 134-137. [CrossRef] [PubMed]

2. Festa, E.; Fretz, J.; Berry, R.; Schmidt, B.; Rodeheffer, M.; Horowitz, M.; Horsley, V. Adipocyte lineage cells contribute to the skin stem cell niche to drive hair cycling. Cell 2011, 146, 761-771. [CrossRef] [PubMed]

3. Jeong, Y.M.; Sung, Y.K.; Kim, W.K.; Kim, J.H.; Kwack, M.H.; Yoon, I.; Kim, D.D.; Sung, J.H. Ultraviolet B preconditioning enhances the hair growth-promoting effects of adipose-derived stem cells via generation of reactive oxygen species. Stem Cells Dev. 2013, 22, 158-168. [CrossRef] [PubMed]

4. Kim, J.H.; Kim, W.K.; Sung, Y.K.; Kwack, M.H.; Song, S.Y.; Choi, J.S.; Park, S.G.; Yi, T.; Lee, H.J.; Kim, D.D.; et al. The molecular mechanism underlying the proliferating and preconditioning effect of vitamin C on adipose-derived stem cells. Stem Cells Dev. 2014, 23, 1364-1376. [CrossRef] [PubMed]

5. Kim, J.H.; Park, S.G.; Kim, W.K.; Song, S.U.; Sung, J.H. Functional regulation of adipose-derived stem cells by PDGF-D. Stem Cells 2015, 33, 542-556. [CrossRef] [PubMed]

6. Jin, S.E.; Sung, J.H. Hair regeneration using adipose-derived stem cells. Histol. Histopathol. 2016, 31, $249-256$. [PubMed]

7. Perez-Meza, D.; Ziering, C.; Sforza, M.; Krishnan, G.; Ball, E.; Daniels, E. Hair follicle growth by stromal vascular fraction-enhanced adipose transplantation in baldness. Stem Cells Cloning Adv. Appl. 2017, 10, 1-10. [CrossRef] [PubMed]

8. Yang, Y.; Choi, H.; Seon, M.; Cho, D.; Bang, S.I. LL-37 stimulates the functions of adipose-derived stromal/stem cells via early growth response 1 and the MAPK pathway. Stem Cell Res. Ther. 2016, 7, 58. [CrossRef] [PubMed]

9. Buhl, A.E.; Waldon, D.J.; Kawabe, T.T.; Holland, J.M. Minoxidil stimulates mouse vibrissae follicles in organ culture. J. Dermatol. Sci. 1989, 92, 315-320. [CrossRef]

10. Headington, J.T. Hair follicle biology and topical minoxidil: Possible mechanisms of action. Dermatologica 1987, 175, 19-22. [CrossRef] [PubMed]

11. Michelet, J.F.; Commo, S.; Billoni, N.; Mahe, Y.F.; Bernard, B.A. Activation of cytoprotective prostaglandin synthase- 1 by minoxidil as a possible explanation for its hair growth-stimulating effect. J. Investig. Dermatol. 1997, 108, 205-209. [CrossRef] [PubMed]

12. Li, M.; Marubayashi, A.; Nakaya, Y.; Fukui, K.; Arase, S. Minoxidil-induced hair growth is mediated by adenosine in cultured dermal papilla cells: Possible involvement of sulfonylurea receptor $2 \mathrm{~B}$ as a target of minoxidil. J. Investig. Dermatol. 2001, 117, 1594-1600. [PubMed]

13. Kwack, M.H.; Kang, B.M.; Kim, M.K.; Kim, J.C.; Sung, Y.K. Minoxidil activates $\beta$-catenin pathway in human dermal papilla cells: A possible explanation for its anagen prolongation effect. J. Dermatol. Sci. 2011, 62, 154-159. [CrossRef] [PubMed]

14. Otomo, S. Hair growth effect of minoxidil. Nihon Yakurigaku Zasshi. Folia Pharmacol. Jpn. 2002, 119, $167-174$. [CrossRef]

15. Han, J.H.; Kwon, O.S.; Chung, J.H.; Cho, K.H.; Eun, H.C.; Kim, K.H. Effect of minoxidil on proliferation and apoptosis in dermal papilla cells of human hair follicle. J. Dermatol. Sci. 2004, 34, 91-98. [CrossRef] [PubMed]

16. Hsu, C.L.; Liu, J.S.; Lin, A.C.; Yang, C.H.; Chung, W.H.; Wu, W.G. Minoxidil may suppress androgen receptor-related functions. Oncotarget 2014, 5, 2187-2197. [CrossRef] [PubMed]

17. Cho, M.K.; Ahn, S.C.; Kim, D.H.; Yu, H.S. Parasite excretory-secretory proteins elicit TRIF dependent CXCL1 and IL-6 mediated allergic inflammation. Parasite Immunol. 2010, 32, 354-360. [CrossRef] [PubMed]

18. Roche, J.K.; Keepers, T.R.; Gross, L.K.; Seaner, R.M.; Obrig, T.G. CXCL1/KC and CXCL2/MIP-2 are critical effectors and potential targets for therapy of Escherichia coli O157:H7-associated renal inflammation. Am. J. Pathol. 2007, 170, 526-537. [CrossRef] [PubMed]

19. De Filippo, K.; Dudeck, A.; Hasenberg, M.; Nye, E.; van Rooijen, K.; Hartmann, M.; Gunzer, A.; Roers, N.H. Mast cell and macrophage chemokines CXCL1/CXCL2 control the early stage of neutrophil recruitment during tissue inflammation. Blood 2013, 121, 4930-4937. [CrossRef] [PubMed]

20. Fujimoto, J.; Sakaguchi, H.; Hirose, R.; Tamaya, T. Expression of platelet-derived endothelial cell growth factor (PD-ECGF) related to angiogenesis in ovarian endometriosis. J. Clin. Endocrinol. Metab. 1999, 84, 359-362. [CrossRef] [PubMed] 
21. Greisler, H.P.; Klosak, J.J.; Dennis, J.W.; Karesh, S.M.; Ellinger, J.; Kim, D.U. Biomaterial pretreatment with ECGF to augment endothelial cell proliferation. J. Vasc. Surg. 1987, 5, 393-399. [CrossRef]

22. Santhosh Kumar, T.R.; Krishnan, L.K. Endothelial cell growth factor (ECGF) enmeshed with fibrin matrix enhances proliferation of EC in vitro. Biomaterials 2001, 22, 2769-2776. [CrossRef]

23. Rezza, A.; Sennett, R.; Tanguy, M.; Clavel, C.; Rendl, M. PDGF signalling in the dermis and in dermal condensates is dispensable for hair follicle induction and formation. Exp. Dermatol. 2015, 24, 468-470. [CrossRef] [PubMed]

24. Tomita, Y.; Akiyama, M.; Shimizu, H. PDGF isoforms induce and maintain anagen phase of murine hair follicles. J. Dermatol. Sci. 2006, 43, 105-115. [CrossRef] [PubMed]

25. Wang, Y.; Crisostomo, P.R.; Wang, M.; Markel, T.A.; Novotny, N.M.; Meldrum, D.R. TGF- $\alpha$ increases human mesenchymal stem cell-secreted VEGF by MEK- and PI3-K- but not JNK- or ERK-dependent mechanisms. Am. J. Physiol. Regul. Integr. Comp. Physiol. 2008, 295, R1115-R1123. [CrossRef] [PubMed]

26. Wang, Y.; Wang, M.; Abarbanell, A.M.; Weil, B.R.; Herrmann, J.L.; Tan, J.; Novotny, N.M.; Coffey, A.C.; Meldrum, D.R. MEK mediates the novel cross talk between TNFR2 and TGF-EGFR in enhancing vascular endothelial growth factor (VEGF) secretion from human mesenchymal stem cells. Surgery 2009, 146, 198-205. [CrossRef] [PubMed]

27. Wang, Y.; Weil, B.R.; Herrmann, J.L.; Abarbanell, A.M.; Tan, J.; Markel, T.A.; Kelly, M.L.; Meldrum, D.R. MEK, p38, and PI-3K mediate cross talk between EGFR and TNFR in enhancing hepatocyte growth factor production from human mesenchymal stem cells. Am. J. Physiol. Cell Physiol. 2009, 297, C1284-C1293. [CrossRef] [PubMed]

28. Crisostomo, P.R.; Wang, Y.; Markel, T.A.; Wang, M.; Lahm, T.; Meldrum, D.R. Human mesenchymal stem cells stimulated by TNF- $\alpha$, LPS, or hypoxia produce growth factors by an NFkB- but not JNK-dependent mechanism. Am. J. Physiol. Cell Physiol. 2008, 294, C675-682. [CrossRef] [PubMed]

29. Kwon, O.S.; Pyo, H.K.; Oh, Y.J.; Han, J.H.; Lee, S.R.; Chung, J.H.; Eun, H.C.; Kim, K.H. Promotive effect of minoxidil combined with all-trans retinoic acid (tretinoin) on human hair growth in vitro. J. Korean Med. Sci. 2007, 22, 283-289. [CrossRef] [PubMed]

30. Kim, W.S.; Park, B.S.; Sung, J.H.; Yang, J.M.; Park, S.B.; Kwak, S.J.; Park, J.S. Wound healing effect of adipose-derived stem cells: A critical role of secretory factors on human dermal fibroblasts. J. Dermatol. Sci. 2007, 48, 15-24. [CrossRef] [PubMed]

31. Yi, T.; Kim, W.K.; Choi, J.S.; Song, S.Y.; Han, J.; Kim, J.H.; Kim, W.S.; Park, S.G.; Lee, H.J.; Cho, Y.K.; et al. Isolation of adipose-derived stem cells by using a subfractionation culturing method. Expert Opin. Biol. Ther. 2014, 14, 1551-1560. [CrossRef] [PubMed]

32. Kim, J.H.; Park, S.G.; Song, S.Y.; Kim, J.K.; Sung, J.H. Reactive oxygen species-responsive miR-210 regulates proliferation and migration of adiposederived stem cells via PTPN. Cell Death Dis. 2013, 4, e588. [CrossRef] [PubMed]

33. Kim, J.H.; Kim, S.H.; Song, S.Y.; Kim, W.S.; Song, S.U.; Yi, T.; Jeon, M.S.; Chung, H.M.; Xia, Y.; Sung, J.H. Hypoxia induces adipocyte differentiation of adiposederived stem cells by triggering reactive oxygen species generation. Cell Biol. Int. 2014, 38, 32-40. [CrossRef] [PubMed]

34. Song, S.U.; Kim, C.S.; Yoon, S.P.; Kim, S.K.; Lee, M.H.; Kang, J.S.; Choi, G.S.; Moon, S.H.; Choi, M.S.; Cho, Y.K.; et al. Variations of clonal marrow stem cell lines established from human bone marrow in surface epitopes, differentiation potential, gene expression, and cytokine secretion. Stem Cells Dev. 2008, 17, 451-461. [CrossRef] [PubMed]

35. Sung, J.H.; An, H.S.; Jeong, J.H.; Shin, S.; Song, S.Y. Megestrol Acetate Increases the Proliferation, Migration, and Adipogenic Differentiation of Adipose-Derived Stem Cells via Glucocorticoid Receptor. Stem Cells Transl. Med. 2015, 4, 789-799. [CrossRef] [PubMed]

(C) 2018 by the authors. Licensee MDPI, Basel, Switzerland. This article is an open access article distributed under the terms and conditions of the Creative Commons Attribution (CC BY) license (http://creativecommons.org/licenses/by/4.0/). 